\title{
De la déconnexion aux TIC comme forme de résistance à l'urgence
}

\section{Francis Jauréguiberry}

\section{(2) OpenEdition}

1 Journals

Édition électronique

URL : http://journals.openedition.org/communicationorganisation/3409

DOI : 10.4000/communicationorganisation.3409

ISSN : 1775-3546

Éditeur

Presses universitaires de Bordeaux

\section{Édition imprimée}

Date de publication : 1 juin 2006

Pagination : 186-195

ISSN : 1168-5549

\section{Référence électronique}

Francis Jauréguiberry, « De la déconnexion aux TIC comme forme de résistance à l'urgence », Communication et organisation [En ligne], 29 | 2006, mis en ligne le 21 juin 2012, consulté le 23 décembre 2020. URL : http://journals.openedition.org/communicationorganisation/3409 ; DOI : https://doi.org/10.4000/communicationorganisation.3409

Ce document a été généré automatiquement le 23 décembre 2020.

(C) Presses universitaires de Bordeaux 


\title{
De la déconnexion aux TIC comme forme de résistance à l'urgence
}

\author{
Francis Jauréguiberry
}

\section{L'urgence comme piège du temps}

1 Ce ne sont pas les technologies de l'information et de la communication (TIC) qui créent l'urgence. Aucune technologie, quelle qu'elle soit, n'agit aussi directement sur les conduites. Les TIC ne sont pas des «arrosoirs » comportementaux dont l'eau serait puisée en dehors du social, de la même façon que les usagers ne sont pas de simples «buvards» qui, selon leur porosité, absorberaient avec plus ou moins de talent l'innovation technologique. Seul un détour par les logiques d'action qui à la fois président l'invention de ces technologies et conditionnent leurs usages permet de donner un sens aux conduites qui leur sont associées. Mais, d'un autre côté, affirmer que ces technologies ne sont pour rien dans la nature des comportements observés serait une erreur. En effet, si les TIC ne créent pas l'urgence, ce sont bien elles qui, de plus en plus souvent, la permettent. C'est parce qu'il y a désormais, grâce à ces technologies, possibilité de réagir immédiatement et jusqu'au dernier moment que de plus en plus d'individus s'inscrivent dans des scénarios limites. Ils calculent en effet toujours plus juste sachant que, si ça ne passe pas, il y aura toujours le recours ultime d'un «appel urgent" pour "réparer » leur retard, de la même façon que certains alpinistes, assurés qu'ils pourront immédiatement déclancher des secours en cas d'accident, s'engagent dans des voies manifestement trop dures pour eux. Ce type d'appel ne devient heureusement pas la règle, mais le raccourcissement des délais et la prise de risques, oui.

2 Cette notion de scénario limite permet de donner une définition de l'urgence. L'urgence naît en effet toujours d'une double prise de conscience: d'une part, qu'un pan incontournable de la réalité relève d'un scénario aux conséquences dramatiques ou inacceptables et, d'autre part, que seule une action d'une exceptionnelle rapidité peut empêcher ce scénario d'aller à son terme. L'une ne va pas sans l'autre, ou alors il ne 
s'agit plus d'urgence. Ce n'est pas parce qu'une action est exceptionnellement rapide qu'elle est urgente. Par exemple, les courses (compétitions) sont par définition toujours rapides, mais rarement urgentes. De même, ce n'est pas parce qu'un scénario catastrophe est en marche qu'il génère ipso facto de l'urgence : le laps de temps laissé à la réaction peut être suffisamment long pour que celle-ci ne soit pas vécue sous la forme d'une rapidité imposée. Cette double prise de conscience déclenche un compte à rebours qu'il s'agit d'arrêter illico presto. Dit autrement : on court à la catastrophe si rien n'est immédiatement mis en œuvre. Le scénario à la base de l'urgence est toujours menaçant, néfaste ou fatal. S'il n'était pas appréhendé comme l'irréversibilité d'une négativité mais d'une positivité, il s'agirait non plus d'urgence mais d'attente ou d'impatience, le « vivement » remplaçant en la matière le « vite ».

3 L'urgence apparait donc comme un piège du temps. La dangerosité de ce piège dépend de l'interprétation qui en est faite, puisque l'urgence naît toujours de la double prise de conscience qui vient d'être évoquée. Les notions de danger et de rapidité étant relatives, il s'ensuit que ce qui est urgent pour certains ne l'est pas pour d'autres. Tout dépend du système de référence des acteurs, de leur subjectivité et de leurs capacités cognitives. Mais, quelles que soient les disparités que l'on peut observer entre individus, la ligne de l'urgence est toujours franchie lorsque la notion de piège du temps apparait clairement. Or, force est de reconnaitre que, globalement, l'immédiateté télécommunicationnelle est en train d'accroître la surface de cette notion. En quelques années seulement (fin du $\mathrm{XX}^{\mathrm{e}}$ ), le processus d'accélération et de densification du temps, à l'œuvre depuis deux siècles, s'est en effet vu doublé par une nouvelle donnée: celle que les informaticiens ont nommée temps réel, synonyme d'immédiateté et de simultanéité. À peine remises des bouleversements que la rationalisation et l'instrumentalisation du temps ont opérés en leur sein, nos sociétés sont confrontées à un nouvel impératif: il leur faut désormais réagir à l'instant. Un espace sans distance et un temps sans délais se superposent peu à peu à l'espace-temps " classique ».

4 Cette nouvelle donne peut être vécue de façon positive: comme multiplicateur d'activités et d'opportunités, comme révélateur de certaines potentialités organisationnelles jusqu'alors inexploitées, comme agent de simplification ou encore comme réhabilitation de l'intuition individuelle dans la gestion des affaires. Elle peut aussi être source de satisfactions, en particulier pour certains professionnels indépendants qui, vivant cette course au temps sous la forme de défis renouvelés, la trouvent passionnante et parfois gratifiante. Mais cette accélération peut aussi donner le vertige, et la chute n'est alors pas exclue. Dans un monde où tout s'accélère et se bouscule, l'individu «branché » (aux TIC), placé en état d'urgence quasi permanent court en effet deux risques.

\section{Les risques de l'urgence}

5 Le premier de ces risques est de réagir à l'impulsion afin d'éviter ce qu'on pourrait appeler "l'effet bouchon": l'accumulation incontrôlable d'informations interdisant leur traitement efficace. Face à une pile de notes, à une succession d'e-mails urgents et à un sans-fin de sollicitations téléphoniques, il faut aller vite. Non seulement il faut toujours être connecté, mais il faut aussi pouvoir répondre rapidement. Dans cette accélération, synonyme de diminution du temps de réflexion, l'accessoire risque de 
recouvrir l'essentiel. Outre le stress lié à l'activité fébrile qui en résulte, le danger d'une telle réaction est de voir remplacer la réflexion et l'imaginaire par une espèce de réflexe à parer au plus pressé. Le branché se convertit en pompier cherchant à éteindre le feu de l'urgence là où il prend. Le coup de fil a dès lors priorité sur la personne présente, le courrier électronique sur le courrier postal, et le beeper arrête tout, séance tenante. Comme si l'individu ou l'organisation se mettaient aux ordres de l'urgence, l'advenant supplantant l'existant.

6 Il est évidemment des situations dans lesquelles nécessité fait loi. Mais l'extension de ce mode de réaction menace de le transformer en une véritable mode de fonctionnement. Focalisées sur la réaction aux sollicitations immédiates, personnes ou structures courent alors le risque de perdre tout pouvoir stratégique au profit de pures tactiques d'adaptation à un environnement qu'elles ne maîtrisent plus. Dans ce cas, l'information devient bruit, la vitesse précipitation, et les passages à l'acte font office de décisions. D'actif et réfléchi, le choix devient réactif et improvisé, et a donc toute chance d'être dépendant. Ce type de conduite risque de rendre obsolète la notion même de projet. Le projet nécessite une certaine confiance dans l'avenir. Il relève d'un « pari » sur le futur en pensant que l'action programmée pourra s'y déployer. Il n'y a évidemment, en la matière, aucune certitude: seule la confiance permet de différer, de planifier, de se représenter ce qui sera contre ce qui est. Mais si le présent lui-même apparaît comme indéterminé, n'est plus vécu que comme immédiateté éphémère, comment penser le projet? Dans un monde où il faut s'adapter rapidement, comment peut-on encore adapter le monde dans la durée ${ }^{1}$ ?

Le second risque pour le branché est de se mettre à hésiter dans l'urgence. Les prises de décision deviennent alors autant de violences que l'individu s'impose dans une situation qu'il ne maîtrise plus. Tensions, stress et parfois même anxiété ont alors toutes chances d'apparaître. Le branché se sent non plus interpellé mais harcelé. Tout un ensemble de pathologies psychosomatiques sont associées à cette contradiction dans laquelle se place l'individu en «surchauffe»occupationnelle ${ }^{2}$. D'un côté la conscience de l'urgence, de l'autre celle de ne pouvoir y faire face, ce qui ne fait qu'augmenter la pression. Il est même des cas où l'individu reste comme "sonné " devant trop d'interpellations. Pour lui, la seule façon de ne pas cesser d'exister complètement, est alors de "craquer ». Entrant dans un véritable état de catalepsie, il "démissionne» par overdose communicationnelle pour tomber dans un vide apathique. Toute "gestion en temps réel » ou tout "pilotage en situation d'urgence » ne saurait évidemment conduire à une telle situation. Non seulement parce que les réactions à l'urgence peuvent avoir été planifiées de façon préalable (on parle alors de "procédures", fruits d'anticipations stratégiques), mais aussi, de façon plus fondamentale, parce que ces réactions peuvent relever d'une rationalité, certes limitée par le temps, mais non hétérogène à une continuité stratégique pourvoyeuse de sens.

Il est évidemment des situations où nécessité fait loi. Mais l'extension de ce mode de réaction menace de le transformer en un véritable mode de fonctionnement. Focalisé sur la réaction aux sollicitations immédiates dont il est l'objet, l'homme pressé court dans ce cas le risque de souffrir de ce que j'ai appelé le «syndrome du branché ». Par syndrome du branché, j'entends l'ensemble des symptômes du mal latent qui guette ceux qui vivent leur expérience d'ubiquité médiatique selon une logique de pure rentabilité au point de s'y faire absorber. C'est tout à la fois l'anxiété du temps perdu, le stress du dernier moment, le désir jamais assouvi d'être ici et ailleurs en même temps, 
la peur de rater quelque chose d'important, l'insatisfaction des choix hâtifs, la hantise de ne pas être branché au bon moment sur le bon réseau, et la confusion due à une surinformation éphémère.

9 Le syndrome du branché, c'est le "mal des télécommunications", la "nausée médiatique» dont est pris le branché lorsqu'il tombe dans ce qu'un de mes informateurs a appelé le «tourbillon ». Comme soumis à l'effet d'une force centrifuge due à une accélération non maîtrisée, l'individu est dépossédé du sens de son action et aspiré par une inflation occupationnelle. Contraint à réagir sur le mode de l'urgence à une masse d'information grandissante et à des aléas de plus en plus fréquents, il se trouve réduit à s'accrocher où il peut s'il ne veut pas sombrer, à «faire des coups » et à développer des tactiques de nature opportuniste. L'acteur est alors moins mû par une logique de type stratégique, visant à adopter les meilleurs moyens par rapport à des fins qu'il s'est fixées, que par une logique de type tactique, visant à s'adapter au mieux à une situation qu'il ne domine plus.

\section{Retrouver le temps}

10 Le tourbillon du branché attire. Il attire parce que la force du courant qui l'alimente est celle du système économique actuel basé sur la généralisation de la gestion en « temps réel » et qu'il est difficile d'échapper à sa pression. Il attire aussi parce que la forme qu'il donne à l'action (la vitesse, le défi, le zapping, le sentiment de puissance) n'est pas sans procurer une certaine jouissance à ceux qui l'expérimentent. Il attire enfin peutêtre parce qu'en ramassant le temps dans une sorte de présent continu, il « distrait » (au sens pascalien du terme) l'individu du temps qui passe, et donc de questions existentielles potentiellement inquiétantes ${ }^{3}$.

11 Mais le tourbillon du branché peut aussi être pensé comme un écueil qu'il s'agit d'éviter. Face à la dispersion et à l'égarement qu'il peut engendrer, à l'aléatoire trop souvent côtoyé et au stress qu'il suscite, à l'éphémère renouvelé dans une sorte d'éternel présent, une réaction apparaît. Elle renvoie à une logique critique qui vise à ne pas se laisser déposséder de sa propre temporalité, de ses propres rythmes au profit d'une mise en synchronie universelle qui unirait « en temps réel » tous les «branchés » $\mathrm{du}$ " grand réseau » dans une sorte de compulsion totalisante. Cette logique critique déroule ce que l'urgence ficelle. Elle résiste à l'idée que la vie ne serait qu'une suite d'instants et d'événements sans liens entre eux. Elle rétablit la duré et replace l'individu dans une continuité qui lui permet de renouer avec un ordre possible de référence. Elle réintroduit l'épaisseur du temps de la maturation, de la réflexion et de la méditation là où le heurt de l'immédiat et de l'urgence oblige à réagir trop souvent sous le mode de l'impulsion. Cette logique est celle de la mise en perspective de soi dans le temps sous la forme d'un récit, d'une narration sans cesse revisitée. Car, en définitive, ce n'est pas le temps qui passe mais l'individu.

12 Le temps que l'on découvre alors est celui du passé, du souvenir et du retour sur soi. C'est aussi le temps de l'anticipation, de la crainte ou de l'espoir. La possibilité de pouvoir joindre immédiatement son interlocuteur pour lui faire part d'une intuition, d'une idée ou d'un élan sentimental est sans doute une chance. Mais elle peut aussi représenter un danger pour l'émotion pensée non plus comme impulsion mais comme tension créatrice. Le risque réside dans le fait de voir l'impulsion chasser l'imagination, et le bavardage remplacer l'échange. Le silence et le différé, condition de retour sur le 
passé et de projection sur l'avenir, sont les complices d'un présent créateur. Mais, lorsque ce présent n'est plus qu'une succession d'immédiats éphémères, où se situe la continuité ? Que reste-t-il en particulier comme trace de l'échange, de la tension vers l'autre et des sentiments que son absence peut susciter? Si Balzac avait pu téléphoner à Madame Hanska ses Lettres à une étrangère auraient-elles seulement été écrites? Pourrions-nous, plus de quatre-vingts ans après, partager l'émotion d'Apollinaire pensant à sa chère Lou s'il avait pu, « en temps réel », lui faire part de son désir et de ses rêveries? Ce temps de la tension créatrice se donne à vivre dans l'arrêt, l'attente, le différé et la mise à distance. Il est non superposable et non susceptible d'être dédoublé : l'individu y contient tout entier.

13 Cette logique critique rappelle que, derrière l'apparence trompeuse d'un temps unique, universel et synchrone (celui des heures de la montre, des jours de l'agenda et des mois du calendrier), le temps est hétérogène. Qu'il n'y a pas un temps, mais des temps. Que la réalité du temps n'est pas seulement le temps réel. Que, face à l'entrée massive de notre société dans une culture de l'immédiat, de l'impulsion et de l'urgence généralisée, il y a des moments qui résistent à l'accélération, des durées qui ne sauraient être brusquées, et des instants qui échappent à la logique du gain et de la vitesse. Ces moments, ces durées et ces instants sont indispensables à la formation de soi comme sujet, c'est-àdire comme acteur capable de construire sa vie de façon autonome. Cette prise de distance en regard de l'instant permet à l'individu de se retrouver et en particulier de vivre une certaine autonomie par rapport à son rôle de gestionnaire efficace que tout le monde (y compris une part de lui-même) s'accorde à lui voir jouer.

\section{L'expérience temporelle du branché}

14 L'expérience du branché dans la gestion du temps est le produit d'une sorte de "dialogue tendu» entre deux logiques d'action, sachant que l'une ne saurait entièrement recouvrir l'autre. D'un côté, une logique de gains et de vitesse qui est celle de la connexion, de la mise en synchronie et de l'urgence. De l'autre, une logique critique qui est celle de l'aménagement d'un temps à soi, de prise de distance et de déconnexion.

Lorsque l'expérience du branché est trop fortement soumise à la logique de gains, de mise en synchronie et de vitesse, elle s'effondre et tombe dans un tourbillon occupationnel. La volonté, lorsqu'elle existe, d'y échapper donne généralement naissance à une conduite de fuite en avant. Voyant qu'il se fait posséder par une accélération non maîtrisée, le "branché dépassé " nourri l'espoir qu'une gestion encore plus rentabiliste de son temps va lui permettre d'échapper à ce tourbillon. Son exaspération communicationnelle se traduit alors souvent par une exacerbation de son désir technique. À l'affût des dernières découvertes, il pense qu'une meilleure couverture du réseau, une plus grande intelligence de transmission, de nouveaux terminaux multifonctions, lui rendront un peu de temps et lui permettront de se retrouver. Soumettant (consciemment ou pas) leur vie à une logique de rentabilisation du temps, ceux qui adoptent cette attitude n'ont souvent pas d'autre choix que cette fuite en avant.

Lorsque l'expérience du branché est, à l'inverse, entièrement dominée par la logique de distanciation, elle conduit à une exigence d'authenticité et souvent à un enfermement subjectiviste dont le «temps à soi » constitue l'horizon. La déconnexion totale aux TIC 
est conduite extrême et très rare. Mais elle existe: sans pour cela se convertir en ermites, ceux qui " se retirent » se renferment sur leur foyer, leur maison, leur jardin... Ils cherchent (je reprends leurs termes) une "nouvelle simplicité", une "vérité de vie », une capacité à « être pleinement là ». Mais la majorité ne peut pas se déconnecter totalement. La pression économique et sociale est tout simplement trop forte. La tentation est alors grande de cliver les communications. D'un côté les communications "rentables", intéressées et stratégiques pour lesquelles la rapidité concourt à leur réussite, et de l'autre les communications "gratuites", intersubjectives et conviviales pour lesquelles le temps n'est pas compté. D'un côté, la communication « utile » et de l'autre la " vraie communication ». Les télécommunications nomades sont associées à la première, dans leur capacité même à organiser la seconde qui, elle se fait (encore ?) en face à face.

La fuite en avant (connexion continue, hyper équipement, abandon de soi dans l'intensité) et le refus (déconnexion totale, burn out) sont des conduites de rupture avec l'expérience du branché. Les deux relèvent finalement d'une impossibilité ou d'une incapacité de conjuguer le désir ou la nécessité d'être «branché » avec la volonté de préserver un temps à soi. Une pleine expérience de «branché » renvoie au contraire à la nature hétérogène des logiques d'action qui la déterminent: utilitaire d'un côté, guidée par un souci de performance, d'efficacité et de gain, et critique de l'autre, motivée par la volonté de ne pas soumettre l'ensemble de sa vie à une mise en synchronie constante.

18 Il s'ensuit des conduites de compromis, de ruse, de fines tactiques permettant de conjuguer au mieux ces deux exigences. Elles consistent pour l'essentiel à instaurer des filtres intelligents entre le réseau (la mise en synchronie) et l'acteur (recul réflexif, temps à soi). Il s'agit de garder le contact sans en devenir l'esclave. Ces filtres sont généralement une tierce personne (en particulier le secrétariat sur le lieux de travail et le conjoint ou les enfants au domicile), un système mécanique de mise en différé (boîte vocale du portable, beeper, répondeur-enregistreur et fax) ou un système de filtre en temps réel (répondeur-enregistreur). Le succès des SMS et du courrier électronique s'inscrit dans cette logique. Une autre forme de mise à distance, que l'on pourrait appeler "repos du guerrier", consiste à se déconnecter de façon régulière mais éphémère afin de se "requinquer». Cette attitude est assez perverse puisqu'elle permet d'encaisser stress et urgence sans pour cela remettre en cause le mode de fonctionnement du système qui les produit. Ce sont par exemple les demi-heures de zen ou de "respirations profondes » organisées par l'entreprise pour ses employés ou de petits stages de déconnexion. " On travaille tous trop, me disant un cadre d'une start up de la Silicon Valley, on est tous workaholics, alors, de temps en temps, on fait des petits stages de désintoxication ». Et l'un des principes essentiels de ces stages consiste précisément à être totalement déconnecté. Tous les ans, le festival Burning man a lieu, durant une semaine, dans le désert du Nevada. En 2005, ils étaient plus de 30000 à passer une semaine sans portable, "déconnectés mais branchés sur tous ceux qu'ils croisaient ». Il est intéressant de noter que ce sont des techniciens, des ingénieurs et des managers qui fréquentent majoritairement ces lieux, avec quelques utopistes, évidemment. Finalement, lorsque la vie devient trop stressante, l'urgence trop pressante, on zappe sur un petit stage de déconnexion pour se recharger...

Bien sûr, l'équilibre est précaire et doit sans cesse être rétabli. Il est des situations ou des périodes où le tourbillon du branché attire plus que d'autres où la volonté critique 
de prise de distance et de préservation d'une temporalité individuelle l'emporte. Mais c'est précisément parce qu'il n'existe en la matière pas de solution définitive que l'on peut parler, à propos de ceux qui se livrent à ce travail de mise en cohérence, d'acteurs sociaux en train de définir un nouvel enjeu social : le droit à la déconnexion.

\section{BIBLIOGRAPHIE}

Aubert N., Le culte de l'urgence. La société malade du temps, Paris, Flammarion, 2003.

Ehrenberg A., L'individu incertain, Paris, Calmann-Lévy, 1995

Jauréguiberry F., Les branchés du portables. Sociologie des usages, Paris, PUF, 2003.

Laïdi Z., Un monde privé de sens, Paris, Fayard, 1994.

Nowotny H., Le temps à soi. Genèse et structuration d'un sentiment du temps, Paris, Maison des sciences de l'homme, 1992.

\section{NOTES}

1. Zaki LAÏDI, 1994, souligne les dangers qu'une telle absorption du futur par le présent représente pour la démocratie. Le politique, pensant renouveler sa légitimation en se mettant à l'écoute de l'immédiat, perd son pouvoir visionnaire producteur de projet pour le futur au profit d'un rôle gestionnaire destiné à surmonter des contraintes du présent. La réduction de la distance entre gestion de l'immédiat et représentation du futur accompagne la chute des catégories d'espoir, d'attente ou d'utopie politique.

2. Sur ce thème, voir Aubert N., 2003.

3. Si l'on accepte en effet, avec Heidegger, que c'est parce qu'il "s'insère dans le temps » (Zeitigung) que l'individu a conscience de son "être-là » (Dasein), et que cette conscience est avant tout celle de sa finitude individuelle (l'être est dans la conscience du temps qui est celle de sa mort), on peut penser que l'écrasement du présent sur l'immédiat est une façon d'échapper à l'angoisse qu'une telle conscience entraîne.

4. Pour une généalogie de cette temporalité individuelle, voir Nowotny H., 1992.

\section{AUTEUR}

\section{FRANCIS JAURÉGUIBERRY}

Mail : francis.jaureguiberry@ 